\title{
Two Forms of Learning following Training to a Single Odorant in Caenorhabditis elegans AWC Neurons
}

\author{
Schreiber Pereira and Derek van der Kooy \\ Department of Molecular Genetics, Faculty of Medicine, and The Donnelly Centre, University of Toronto, Toronto, Ontario M5S 3E1, Canada
}

The nematode Caenorhabditis elegans can adapt to both the AWC-sensed odorants benzaldehyde (Bnz) and isoamyl alcohol (IsoA) and can reciprocally cross-adapt. Yet we reveal that these four adaptation scenarios actually represent two distinct forms of learning: nonassociative habituation and associative learning by pairing with a starvation unconditioned stimulus. Training to the single odorant IsoA leads to both associative and nonassociative memory traces, which can be preferentially accessed by either a Bnz or IsoA retrieval stimulus, respectively. This represents the first demonstration in which the form of learning displayed after training to a single stimulus is a function of the retrieval stimulus used. Furthermore, these two forms of learning can be genetically double dissociated despite both forms occurring within the AWC primary sensory neuron. We find that associative learning requires the cGMP-dependent kinase egl-4 and insulin signaling, which acts downstream of egl-4. In contrast, nonassociative learning requires neither of these genes, but does require the TRPV channel osm-9, which is dispensable for associative learning. In addition, we find that the arrestin arr-1 is promiscuous between associative and nonassociative learning in mediating the adaptive response to the IsoA retrieval stimulus, suggesting that distinct forms of memory may nonetheless use common downstream effectors.

\section{Introduction}

Despite their simplicity, Caenorhabditis elegans are capable of both nonassociative and associative learning (de Bono and Maricq, 2005; Giles and Rankin, 2009). Nonassociative learning involves the modulation of response to a single stimulus that is not due to an inability of the sensory or motor system to respond (fatigue) and is sensitive to dishabituation (Groves and Thompson, 1970). Associative learning involves the modulation of a behavior to a conditioned stimulus (CS) by its predictive pairing with an unconditioned stimulus (US) (Levitan and Kaczmarek, 1991). Worms can pair the odorant diacetyl (CS) with aversive acetic acid (US) (Morrison et al., 1999), the smell of pathogenic bacteria (CS) with sickness (US) (Zhang et al., 2005; Ha et al., 2010), as well as a number of cues such as temperature (CS) (Hedgecock and Russell, 1975; Mori and Ohshima, 1995; Mohri et al., 2005), salt (CS) (Wen et al., 1997; Saeki et al., 2001), or odorants (CS) (Nuttley et al., 2002; Torayama et al., 2007) with its feeding state (US).

Received Aug. 15, 2011; revised March 6, 2012; accepted March 19, 2012.

Author contributions: S.P. and D.v.d.K. designed research; S.P. performed research; S.P. and D.v.d.K. analyzed data; S.P. and D.v.d.K. wrote the paper.

This work was supported by the Natural Sciences and Engineering Research Council of Canada (to D.v.d.K.). We thank all members of the van der Kooy Laboratory worm group, particularly Karen Kok, Susan Runciman, Glenn Wolfe, and especially Chia Hsun Anthony Lin, as well as Brenda Coles-Takabe. We also express our gratitude to the Noelle D. L'Etoile Laboratory at the University of California at Davis, the Yuichi lino Laboratory at the University of Tokyo, and the Caenorhabditis elegans Center at the University of Minnesota, which receives funding from the National Institutes of Health, for providing us with strains.

The authors declare no competing financial interests.

Correspondence should be addressed to Derek van der Kooy, Donnelly Centre for Cellular and Biomolecular Research, University of Toronto, 160 College Street, Toronto, ON M5S 3E1, Canada. E-mail: derek.van.der.Kooy@utoronto.ca.

DOI:10.1523/JNEUROSCI.4221-11.2012

Copyright $\odot 2012$ the authors $\quad 0270-6474 / 12 / 329035-10 \$ 15.00 / 0$
C. elegans sense odors through three amphid sensory neuron pairs: the AWA, AWB, and AWC (Bargmann and Horvitz, 1991; Bargmann et al., 1993). While in the naive state each pair is hardwired for repulsion (AWB) or attraction (AWA and AWC) (Troemel et al., 1997), behavior to many odorants is plastic and exposure to an attractive odorant can lead to adaptation, a generic description for loss of attraction following training (Colbert and Bargmann, 1995). Yet such adaptation could result from habituation or aversive associative learning. Nuttley et al. (2002) demonstrated that adaptation to the initially attractive, AWCsensed odorant benzaldehyde (Bnz) resulted from its association with starvation. Worms can also adapt to another AWC-sensed odorant, isoamyl alcohol (IsoA) (Colbert and Bargmann, 1995). In addition, IsoA and Bnz demonstrate a unique phenomenon of reciprocal cross-adaptation (Colbert and Bargmann, 1995), despite the ability of worms to discriminate between them (Bargmann et al., 1993; Wes and Bargmann, 2001). AtkinsonLeadbeater et al. (2004) used this phenomenon to assign a role to an unknown polymorphism in the CB4856 Hawaiian strain. If worms were conditioned and tested to Bnz (henceforth all adaptation protocols are listed as odorant trained $\rightarrow$ odorant tested), CB4856 displayed a deficit relative to the wild-type N2 strain. However, if worms were tested in $\mathrm{Bnz} \rightarrow \mathrm{IsoA}$ or IsoA $\rightarrow$ IsoA scenarios, no deficit could be seen. As a result, the authors postulated that CB4856 possessed a Bnz-specific memory retrieval deficit.

A number of genes have been implicated in AWC adaptation, including members of the insulin signaling pathway (Chalasani et al., 2010; Lin et al., 2010), the transient receptor potential type $\mathrm{V}$ (TRPV) channel osm-9 (Colbert et al., 1997), the cGMPdependent kinase egl-4 (L'Etoile et al., 2002), the arrestin arr-1 (Palmitessa et al., 2005), and the guanalyl cyclase $g c y$-28 (Tsuno- 
zaki et al., 2008). We therefore mimicked the approach of Atkinson-Leadbeater et al. (2004), instead analyzing candidate genes to assign them to specific components (acquisition or retrieval) or types (habituation or associative learning) of memory.

\section{Materials and Methods}

Strains used. N2 (Bristol strain; the wild type in this study) (Caenorhabditis elegans Center, University of Minnesota, Minneapolis, MN), ins-1(nj32) (gift from Iino Laboratory, University of Tokyo, Tokyo, Japan), ins1 (nr2901) (Caenorhabditis elegans Center, University of Minnesota), daf2(e1370) (gift from lino Laboratory, University of Tokyo), age-1 (hx546) (gift from Iino Laboratory, University of Tokyo), age-1 ( $x \times 546)$;odr-3p::age-1 (gift from Iino Laboratory, University of Tokyo), age-1(hx546);gyc-5p::age-1 (gift from Iino Laboratory, University of Tokyo), osm-9(ky10) (Caenorhabditis elegans Center, University of Minnesota), arr-1 (ok401) (Caenorhabditis elegans Center, University of Minnesota), egl-4(ks60) (Caenorhabditis elegans Center, University of Minnesota), dop-3(ok295) (Caenorhabditis elegans Center, University of Minnesota), N2;odr-3p::GFP::egl-4;odr-1p::RFP (gift from L'Etoile Laboratory, University of California at Davis, Davis, CA), N2::odr-3p:::NLS::GFP:::egl-4 (gift from L'Etoile Laboratory, University of California at Davis), and ins-1 (nr2901);odr-3p::NLS::GFP::egl-4 (created inhouse). All studies were conducted using young adult hermaphrodites of these strains.

Cultivation and general methods. All animals were cultivated on nematode growth medium (NGM) agar plates seeded with Escherichia coli OP50 bacteria (maintenance conditions). Animals were both maintained and tested at $20^{\circ} \mathrm{C}$ as described previously (Nuttley et al., 2002) unless otherwise indicated. Animals were age synchronized using bleach isolation of eggs. Briefly, mixed populations of worms were collected in $1.5 \mathrm{ml}$ of M9 buffer, in a $15 \mathrm{ml}$ Falcon tube, to which $1 \mathrm{ml}$ of sodium hypochlorite (10-15\% available chlorine) (Sigma-Aldrich), $2.5 \mathrm{ml}$ of $1 \mathrm{~N} \mathrm{NaOH}$, and $1 \mathrm{ml}$ of water were added. The mixture was incubated for $5 \mathrm{~min}$ with shaking, after which the tube was filled with M9 to stop the reaction. Eggs were then washed (centrifugation followed by removal of supernatant and filling the tube with new M9) five times before being left overnight in M9 buffer with shaking. The following morning, L1 arrested larvae were then plated on maintenance conditions. Young adult worms (50-54 h after plating) were used for all experiments. For experiments with daf-2 mutants in Figure $4 b$, all animals were cultivated at $16^{\circ} \mathrm{C}$ and tested at $23^{\circ} \mathrm{C}$ (permissive and nonpermissive temperature, respectively) to prevent constitutive dauer formation and perform experimentation in the absence of daf- 2 function. These animals were tested $72 \mathrm{~h}$ after plating to account for slower growth at $16^{\circ} \mathrm{C}$. For rescues of IsoA $\rightarrow \mathrm{Bnz}$ adaptation in Figure $4 d$, for all animals, synchronization was achieved by isolating $\mathrm{L} 1$ larvae using a centrifuge (separation of adults by spinning at $50 \times g$ for 1 $\mathrm{min}$, isolation of L1s by spinning at $150 \times g$ for $1 \mathrm{~min}$ ) and tested $50-54$ $\mathrm{h}$ after plating.

Olfactory adaptation paradigm. Olfactory adaptation was performed as described previously (Nuttley et al., 2002) using either benzaldehyde (Sigma-Aldrich) or isoamyl alcohol (IUPAC: 3-methyl-butanol) (SigmaAldrich). All tests were done using fresh $6 \mathrm{ml}, 23$ inch, 100 mM NGM agar plates. Worms were washed with autoclaved water, plated, and dried with Kimwipes. Two microliters of the training odorant was placed on a piece of Parafilm on the inside of the lid, and the plate was sealed with Parafilm. Training was conducted for $1 \mathrm{~h}$ ( $2 \mathrm{~h}$ for the Iso $\mathrm{A} \rightarrow \mathrm{Bnz}$ protocols), unless otherwise specified. Worms were always tested immediately following training except in the dishabituation experiment in Figure $2 e$ (see below). Ten minutes before testing, a $1 \mu \mathrm{l}$ spot of $1 \mathrm{M}$ sodium azide was placed at the center of each $2 \mathrm{~cm}$ circle on either side of the plate (see Fig. 1). This was done to paralyze worms once they approach either the odorant or counterspot, thereby ensuring that only the worm's initial choice on the testing plate is assayed. To begin testing, worms were again washed off with water in to a $15 \mathrm{ml}$ Falcon tube and allowed to settle, and then $50-200$ animals were placed in the center of a test plate and dried with Kimwipes. One microliter of dilute odorant (1\% Bnz or $0.1 \%$ IsoA dissolved in an ethanol solvent) was placed on the center of one $2 \mathrm{~cm}$ circle and a $1 \mu \mathrm{l}$ of counterspot of ethanol at the center of the other to control for any effect of the ethanol solvent on chemotaxis, both where sodium azide had been placed previously. Plates were sealed with Parafilm and after $1 \mathrm{~h}$ of testing placed at $4^{\circ} \mathrm{C}$ to stop the experiment. Chemotactic indices were calculated based on the following formula: $\mathrm{CI}=$ (number of worms within $2 \mathrm{~cm}$ of odor - number of worms within $2 \mathrm{~cm}$ of counterspot)/total. For butanone dishabituation experiments in Figure $2 e$, animals were trained for $1 \mathrm{~h}$ with IsoA (or starvation only for control) as in Figure $2 c$. For dishabituation groups, after $1 \mathrm{~h}$ the Parafilm with IsoA on the lid was removed and replaced with new Parafilm. Ten microliters of $100 \%$ butanone (Sigma-Aldrich) was placed on the Parafilm and the plates placed over the lid for $5 \mathrm{~min}$. For no dishabituation controls, the procedure was the same (controlled for the delay) except that no butanone was added to the new Parafilm. After $5 \mathrm{~min}$, worms were washed off and tested as described previously. For rescue experiments in Figure $4 d$, only GFP + animals were scored. For analysis of egl-4(ks60) approach to $100 \% \mathrm{Bnz}$, experiments were performed similarly except that no training period was used (naive worms were transferred from maintenance conditions to testing plates). A $1 \mu \mathrm{l}$ spot of $100 \% \mathrm{Bnz}$ was placed at the center of one of the $2 \mathrm{~cm}$ circles and no-ethanol counterspot was used since the Bnz stimulus was not diluted in an ethanol solvent. Again, the experiment was ended after $1 \mathrm{~h}$ of testing.

Use of food or serotonin during training. For food block, $10 \mathrm{ml}$ of LuriaBertani media was inoculated with OP50 E. coli and grown overnight at $37^{\circ} \mathrm{C}$ with shaking. The following morning, bacteria were spun down at $3600 \mathrm{rpm}$ (MSE Mistral 2000 centrifuge) for $5 \mathrm{~min}$, washed with water, and respun to remove residual LB and resuspended with $500 \mu \mathrm{l}$ of water. The bacteria was then spread on to two $6 \mathrm{ml}$ NGM plates and dried in a laminar flow hood (equal to $\sim 10 \mathrm{ml}$ of bacteria at $\mathrm{OD}_{560}=0.5$ per plate). For serotonin, a solution of $0.1 \mathrm{M}$ serotonin creatine sulfate complex (Sigma-Aldrich) was made by dissolving it in water at $70^{\circ} \mathrm{C}$ with occasional shaking, and $800 \mu \mathrm{l}$ of the solution was then plated on to $6 \mathrm{ml}$ training plates and dried. These training plates were then used as described above.

Confocal microscopy. For confocal analysis, young adult hermaphrodite worms were conditioned for $2 \mathrm{~h}$ to each odorant as described above. Animals were then washed off and plated on an agarose pad on a slide, made by adding $2 \%$ agarose and water solution to each slide and drying overnight at room temperature, paralyzed with $1 \mu \mathrm{l}$ of sodium azide, and covered with a coverslip. Slides were imaged immediately on an Olympus Fluoview FV1000 confocal microscope with a $60 \times$ oil-immersion objective and assayed manually (five worms per slide, where $n=6$ slides).

Statistical analysis. All data represent the aggregation of two independent experiments with a combined $n=6$ plates for chemotactic data and $n=30$ worms for confocal analysis. Statistical analysis was performed according to Ryder and Robakiewicz (2001). For all figures, significance $(p<0.05)$ was tested using a one- or two-way ANOVA, as indicated, with post hoc Bonferroni's corrections. One-way ANOVA was chosen for experiments in which only a single strain was assayed in multiple conditions. For all genetic analysis, two-way ANOVAs were selected to look for interactions between strain and condition. For analysis of egl-4 approach to $100 \% \mathrm{Bnz}$, a two-tailed $t$ test was used to test for significance. Analysis was performed using SigmaStat 3.1 and Prism 5.0a software. All reported values are means \pm SEM.

\section{Results}

Only the IsoA $\rightarrow$ IsoA adaptation protocol is nonassociative

Previous work in our laboratory has demonstrated that olfactory adaptation to the odorant benzaldehyde is not merely a habituative response or sensory fatigue, but is dependent on pairing the odorant with starvation (Nuttley et al., 2002). As such, the presence of food (E. coli OP50) or exogenous serotonin, a food signal (Luedtke et al., 2010), obviates the adaptive response in a manner consistent with the properties of associative learning. This suggested a serotonergic mechanism acts on a food-sensitive step in Bnz adaptation to block memory formation (Nuttley et al., 2002). To decipher the pathways involved in adaptation to the two AWC-sensed odorants, Bnz and IsoA, we sought to investigate whether all four adaptation experimental protocols $(\mathrm{Bnz} \rightarrow \mathrm{Bnz}, \mathrm{IsoA} \rightarrow \mathrm{IsoA}, \mathrm{Bnz} \rightarrow \mathrm{IsoA}$, and $\mathrm{IsoA} \rightarrow \mathrm{Bnz})$ 

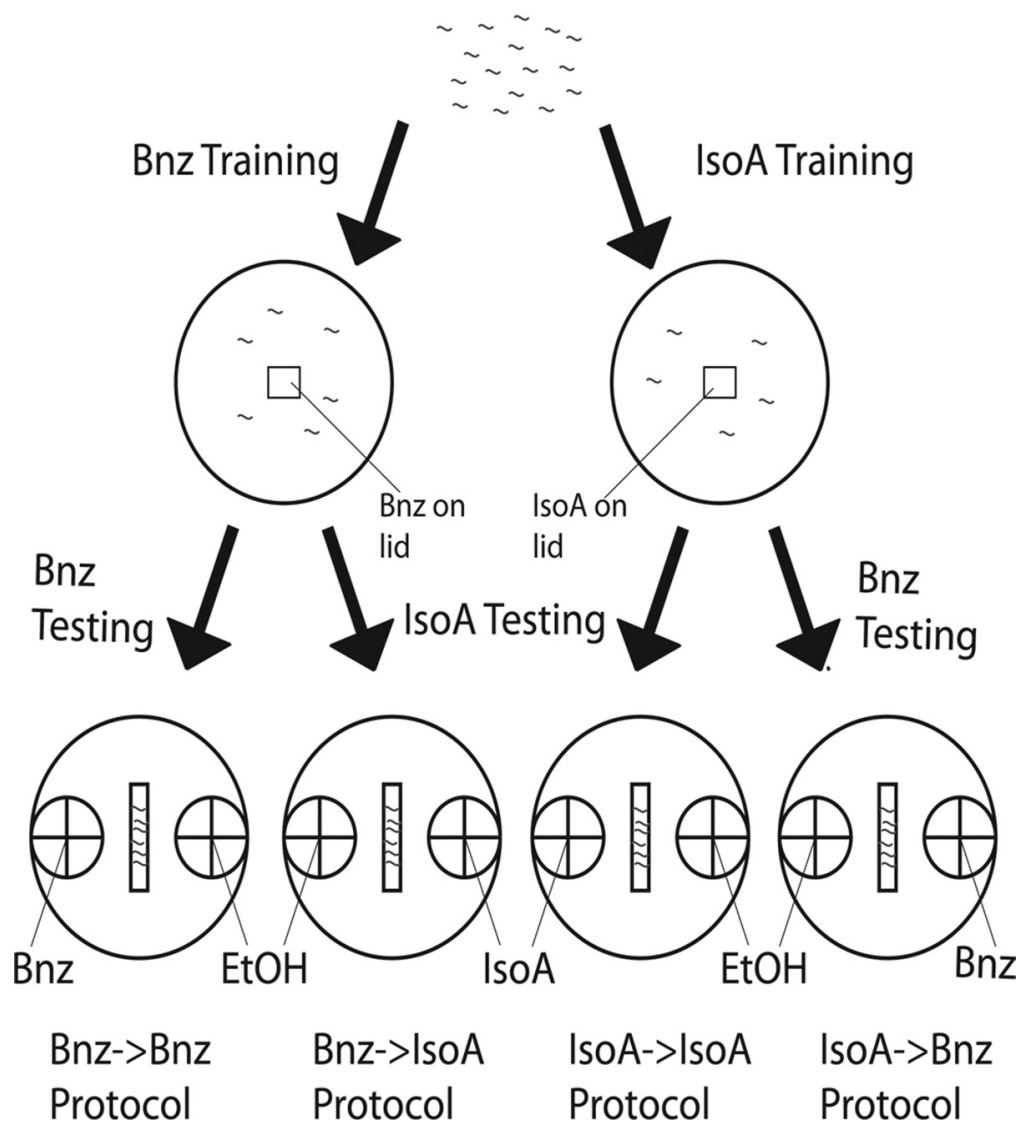

Figure 1. Schematic illustration of the four adaptation experimental protocols. We define four adaptation experimental protocols based simply on the combination of odorant trained and tested under conditions of starvation: $\mathrm{Bnz} \rightarrow \mathrm{Bnz}, \mathrm{Bnz} \rightarrow \mid \mathrm{soA}$, $\mathrm{IsOA} \rightarrow \mathrm{Bnz}$, and IsoA $\rightarrow \mid \mathrm{soO}$ (listed bottom). Each protocol is always accompanied by the appropriate untrained, starvation only (represented by $\emptyset$ ) chemotactic control to the testing odorant (not depicted). Iso $\mathrm{A} \rightarrow \mid \mathrm{s} 0 \mathrm{~A}$ and $\mathrm{Bnz} \rightarrow \mid$ soA are always accompanied with a $\emptyset \rightarrow \mid$ soA control and $\mathrm{Bnz} \rightarrow \mathrm{Bnz}$, and $\mathrm{IsO} A \rightarrow B n z$ are always accompanied by a $\emptyset \rightarrow B n z$ control to ensure that lack of attraction is due to an adapted response, not a lack of attraction in untrained worms to begin with. Worms (gray squiggles) are washed off maintenance conditions and plated on $6 \mathrm{ml} \mathrm{NGM}$ agar training plates without food ( 2 top circles), each of which have $2 \mu \mathrm{l}$ of training odorant, $\mathrm{Bnz}$ (left) or IsoA (right), on a piece of Parafilm (square) on the lid. After drying with Kimwipes, worms are trained for $1 \mathrm{~h}$ ( 3 left protocols) or $2 \mathrm{~h}$ (right protocol) for Iso $\rightarrow$ Bnz. Ten minutes before testing, $1 \mu \mathrm{l}$ of $1 \mathrm{~m}$ sodium azide is added to the center of each $2 \mathrm{~cm}$ circle on the sides of the agar testing plates (bottom 4 circles) to paralyze worms after an initial approach to either spot. Worms are washed off training plates and plated in the center (rectangle) of each testing plate and dried. On each testing plate, a retrieval stimulus of either $1 \% \mathrm{Bnz}$ (first and fourth protocols from left) or $0.1 \% \mathrm{Is} 0 \mathrm{~A}$ (second and third protocols from left) in an ethanol solvent is added to the center of one of the circles. On every plate, a counterspot of ethanol is added to control for the solvent. Worms are able to migrate on the plate for $1 \mathrm{~h}$. Chemotatic index (Cl) is calculated according to the following formula: $\mathrm{Cl}=$ (number of worms within odorant circle - number of worms within ethanol circle)/total number of worms on the test plate. Figures 2-6 are analyses of the sensitivity of each of the four protocols to a manipulation (always accompanied by analysis of the effect of the manipulation on the corresponding starvation only approach as well). Simply, the manipulations are the presence of food during training (Fig. 2), the presence of serotonin during training (Fig. 3), mutation of the insulin signaling pathway (Fig. 4), mutation of candidate nonassociative learning genes (Fig. 5), and mutation (hypomorphic and hypermorphic) of egl-4 (Fig. 6). The sensitivity of each protocol to a manipulation allows us to define whether that protocol is associative or nonassociative and what genes its adaptation mechanism uses.

(Fig. 1) of the two odorants were dependent on pairing the odor with starvation. Atkinson-Leadbeater et al. (2004) had demonstrated that, in addition to the $\mathrm{Bnz} \rightarrow \mathrm{Bnz}$ protocol, $\mathrm{Bnz} \rightarrow$ IsoA adaptation could also be obviated by food during training. Figure 2 displays the effect of food during training on each adaptation permutation. Note that, due to the weak baseline adaptive response of IsoA $\rightarrow \mathrm{Bnz}$, it became problematic to see a significant food/serotonin block effect (data not shown). Thus, the training period was increased to $2 \mathrm{~h}$ for all studies with IsoA $\rightarrow \mathrm{Bnz}$, although appropriate controls negate an effect of training period as an alternative explanation of the data (see below). Consistent with the results of Atkinson-
Leadbeater et al. (2004), food during training blocked the adaptive response in the case of $\mathrm{Bnz} \rightarrow \mathrm{Bnz}$ and $\mathrm{Bnz} \rightarrow \mathrm{IsoA}$ adaptation (Fig. $2 a, b$ ). In addition, food was also shown to block IsoA $\rightarrow$ Bnz adaptation (Fig. 2d). Surprisingly, only the $\mathrm{IsoA} \rightarrow$ IsoA adaptation protocol failed to demonstrate any sensitivity to the presence of food during training (Fig. 2c).

To discount any effect of training period due to the increased training period of the Iso $\mathrm{A} \rightarrow \mathrm{Bnz}$ protocol $(2 \mathrm{~h}$ rather than $1 \mathrm{~h}$ for all others), all three other experimental protocols were repeated with $2 \mathrm{~h}$ of training, and again only the IsoA $\rightarrow$ IsoA protocol did not display any evidence of a food block effect (data not shown). Yet it remained possible that the IsoA $\rightarrow$ IsoA adaptation was actually a form of sensory or motor fatigue rather than true habituation (Groves and Thompson, 1970), and we therefore asked whether this form of adaptation was sensitive to dishabituation. In addition to $\mathrm{Bnz}$ and IsoA, the AWC sensory neurons also sense the odorant butanone (Bargmann et al., 1993), which, however, does not display cross-adaptation with either Bnz or IsoA (Colbert and Bargmann, 1995). We therefore postulated that a strong butanone stimulus could act as a potent dishabituation stimulus targeting the AWC. Indeed, we found that a strong pulse of butanone $(10 \mu \mathrm{l}$ of $100 \%$ butanone on the agar plate lid for 5 min) was able to significantly rescue approach to IsoA (Fig. 2e). Given that the IsoA $\rightarrow$ IsoA adaptation is sensitive to dishabituation, this argues that it represents a form of nonassociative learning rather than sensory or motor fatigue. When the adaptation protocols were tested for their sensitivity to exogenous serotonin, rather than food, similar results were found (Fig. 3). Serotonin was found to block the IsoA $\rightarrow \mathrm{Bnz}$ but not the IsoA $\rightarrow$ IsoA protocol (Fig. $3 c, d$ ). Once more, the inability to block the Iso $\mathrm{A} \rightarrow$ IsoA, but not the Iso $\rightarrow$ Bnz adaptation was not a function of training period as a $2 \mathrm{~h} \mathrm{Iso} \rightarrow$ IsoA protocol was still insensitive to serotonin (Fig. $3 e$ ).

The results therefore have the startling implication that while the $\mathrm{Bnz} \rightarrow \mathrm{Bnz}$ and the two crossadaptation protocols ( $\mathrm{Bnz} \rightarrow \mathrm{IsoA}$ and IsoA $\rightarrow \mathrm{Bnz}$ ) are dependent on a memory that associates starvation with the odor and can be blocked by a serotonergic mechanism, the adapted response seen in the IsoA $\rightarrow$ IsoA protocol is a form of nonassociative learning that is independent of these traits. Given that both the Iso $\mathrm{A} \rightarrow$ Iso $\mathrm{A}$ and Iso $\mathrm{A} \rightarrow \mathrm{Bnz}$ protocol employ training to the same stimulus, this remarkably implies that the single odorant IsoA is able to generate two distinct forms of memory after training and, furthermore, that we can preferentially sample either associative or nonassociative memory by using Bnz or IsoA as the retrieval stimulus, respectively. 

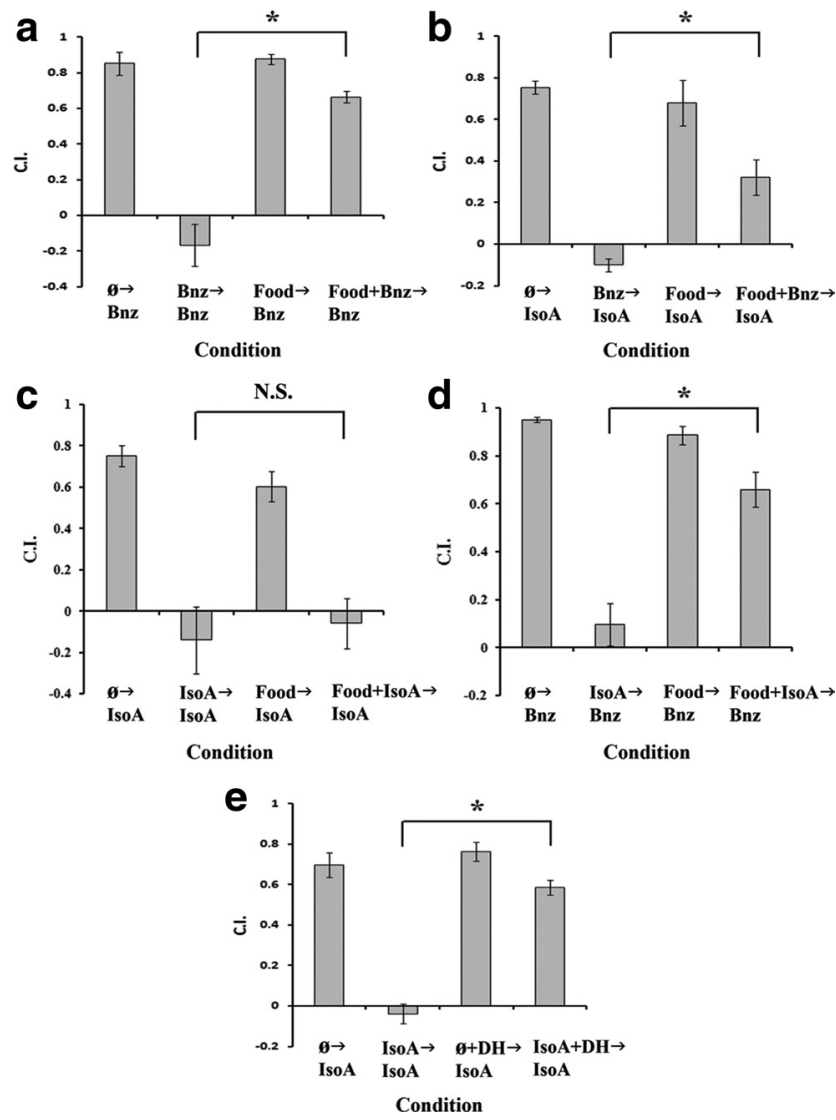

Figure 2. Only the $\mathrm{I} \mathrm{s}_{\mathrm{A}} \mathrm{A} \rightarrow \mathrm{I} \mathrm{s} 0 \mathrm{~A}$ protocol does not dependent on a starvation unconditioned stimulus and is therefore not a form of associative learning. For all panels, data shown are mean \pm SEM, and an asterisk indicates a significant difference between condition $\left({ }^{*} p<0.05\right.$ by Bonferroni's $t$ test), whereas N.S. indicates not significant. $n=6$ plates for all conditions. For $\boldsymbol{a}-\boldsymbol{d}$, N2 wild-type worms were tested in conditions from left to right as follows (with the corresponding associative learning conditions listed in brackets): approach to indicated odorant after starvation (US only), approach to odorant after training with specified odorant during starvation (CS + US), approach to odorant after an hour with food and no training (no US, no CS), and approach to odorant after training in the presence of food (CS only). $\boldsymbol{a}$, Worms were tested for their approach to Bnz using Bnz as the training stimulus ( $B n z \rightarrow B n z$ ). From left to right, columns are as follows: approach to Bnz after starvation ( $\emptyset \rightarrow B n z)$, starvation with Bnz training $(\mathrm{Bnz} \rightarrow \mathrm{Bnz}$ ), food with no training (Food $\rightarrow \mathrm{Bnz}$ ), and training with food (Food $+\mathrm{Bnz} \rightarrow \mathrm{Bnz}$ ). A one-way ANOVA revealed a significant effect of condition $\left(F_{(3,20)}=49.256 ; p<0.001\right)$. A Bonferroni $t$ test indicated a significant effect of food on adaptation $(\mathrm{Bnz} \rightarrow \mathrm{Bnz}$ vs Food $+B n z \rightarrow B n z)$. $\boldsymbol{b}$, Animals were tested as before in the $\mathrm{Bnz} \rightarrow \mathrm{IsoA}$ protocol. A one-way ANOVA revealed a significant effect of condition $\left(F_{(3,20)}=28.540 ; p<0.001\right)$. A significant effect of food on adaptation ( $\mathrm{Bnz} \rightarrow \mid \mathrm{soA}$ vs $\mathrm{Food}+\mathrm{Bnz} \rightarrow \mid \mathrm{soA}$ ) was found. $c$, Animals were tested in the I $\mathrm{SO} \rightarrow \rightarrow \mathrm{S} O \mathrm{~A}$ protocol. A one-way ANOVA revealed a significant effect of condition $\left(F_{(3,20)}=16.934 ; p<0.001\right)$, but a Bonferroni $t$ test found no significant effect of food on

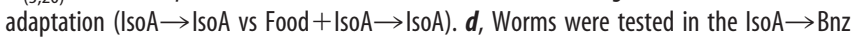
protocol, a one-way ANOVA revealed a significant effect of condition $\left(F_{(3,20)}=41.43 ; p<\right.$ 0.001 ), and again a Bonferronit test found a significant effect of food on training (Iso $A \rightarrow B n z$ vs Food $+\mathrm{IsOA} \rightarrow \mathrm{Bnz})$. $\boldsymbol{e}$, Adaptation in the IsoA $\rightarrow \mid \mathrm{s} 0 \mathrm{~A}$ protocol is sensitive to a butanone dishabituation stimulus. Animals were tested for their chemotaxis to IsoA following either, from left to right, starvation $(\emptyset \rightarrow \mid \mathrm{s} O \mathrm{~A})$, IsoA training $(\mathrm{IsOA} \rightarrow \mid \mathrm{s} 0 \mathrm{~A})$, starvation followed by butanone dishabituation $(\emptyset+\mathrm{DH} \rightarrow \mid \mathrm{s} 0 \mathrm{~A})$, and IsoA training followed by butanone dishabituation (IsoA $+\mathrm{DH} \rightarrow \mid \mathrm{s} 0 \mathrm{~A})$. A one-way ANOVA revealed a significant effect of condition $\left(F_{(3,20)}=\right.$ 55.87; $p<0.05)$, and a Bonferroni $t$ test showed a significant difference between trained animals with or without presentation of the butanone dishabituation stimulus.

\section{Insulin signaling is only required in associative learning protocols}

We next sought to investigate whether particular genes known to play a role in AWC adaptation could be localized to specific components (acquisition vs retrieval) and types (associative vs non-
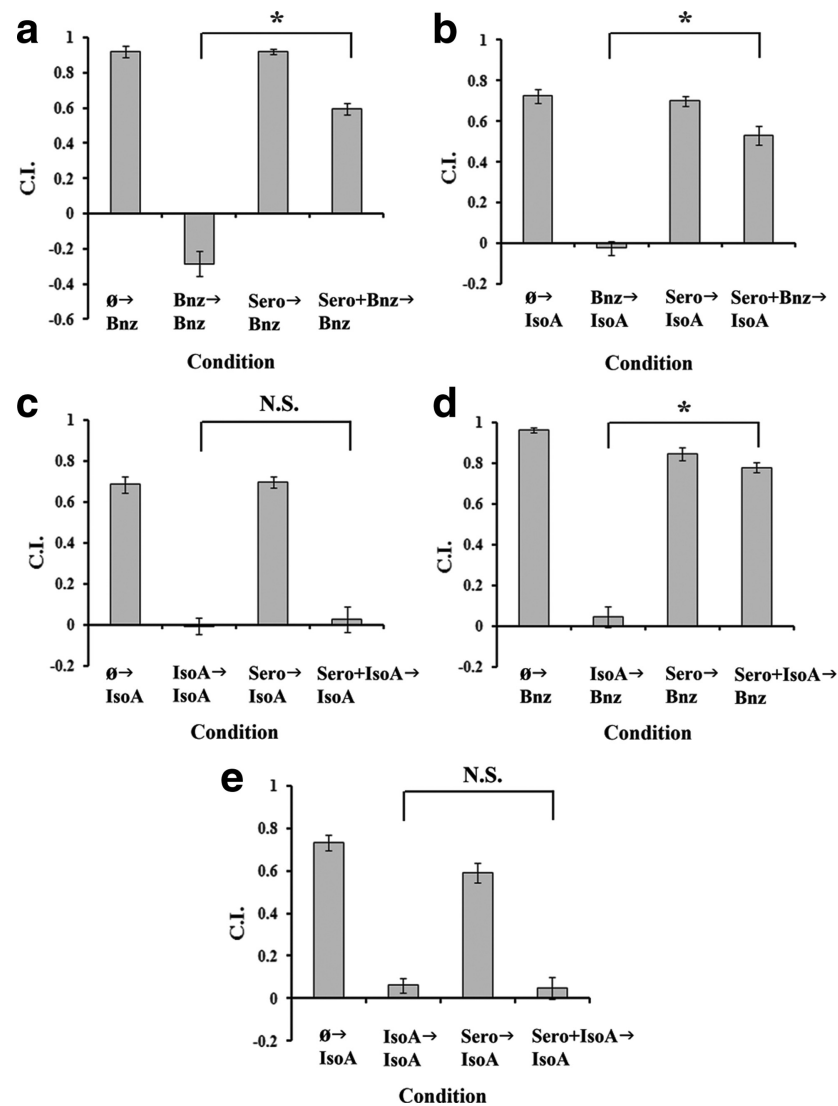

Figure 3. Only the $\left|s_{0} \mathrm{~A} \rightarrow\right| \mathrm{s} O \mathrm{~A}$ protocol is insensitive to the food signal serotonin and sensitivity is not a function of training period. For all panels, data shown are mean \pm SEM; an asterisk indicates significant difference between condition ( ${ }^{*} p<0.05$ by Bonferroni's $t$ test), whereas N.S. indicates not significant. $n=6$ plates for all conditions. Conditions were as described in Figure 2 except replacing food with exogenous serotonin. $\boldsymbol{a}$, Worms were tested in the $\mathrm{Bnz} \rightarrow \mathrm{Bnz}$ permutation. A one-way ANOVA revealed a significant effect of condition $\left(F_{(3,20)}=175.723 ; p<0.001\right)$. A Bonferroni $t$ test indicated a significant effect of food on training (Bnz $\rightarrow$ Bnz vs Sero $+B n z \rightarrow B n z$ ). $\boldsymbol{b}$, Animals were tested as before in the $B n z \rightarrow \mid s 0 A$ permutation. A one-way ANOVA revealed a significant effect of adaptation $\left(F_{(3,20)}=92.270\right.$; $p<0.001)$. A significant effect of food on training ( $\mathrm{Bnz} \rightarrow \mid \mathrm{so} \mathrm{A}$ vs Sero $+\mathrm{Bnz} \rightarrow \mid \mathrm{so} \mathrm{A})$ was revealed. c, Worms were tested in the $I s 0 \mathrm{~A} \rightarrow \mid \mathrm{s} 0 \mathrm{~A}$ protocol. A one-way ANOVA revealed a significant effect of condition $\left(F_{(3,20)}=79.060 ; p<0.001\right)$, but a Bonferroni $t$ test found no significant effect of food on training ( $I s 0 A \rightarrow \mid s 0 A$ vs Sero $\left.+\left|s_{0} A \rightarrow\right| s 0 A\right)$. d, Animals were tested in IsOA $\rightarrow$ Bnz permutation, a one-way ANOVA revealed a significant effect of condition $\left(F_{(3,20)}=151.738 ; p<0.001\right)$ and also found a significant effect of food on training $(\mathrm{Is} \mathrm{A} \rightarrow \mathrm{Bnz}$ vs Sero $+\mathrm{Is} 0 \mathrm{~A} \rightarrow \mathrm{Bnz}) . \boldsymbol{e}$, To ensure the difference in serotonin sensitivity between the $\mathrm{IsOA} \rightarrow \mathrm{I} O \mathrm{SO}$ and $\mathrm{IsO} \rightarrow \mathrm{Bnz}$ experimental protocols was not an artifact of the increased training period used in the latter, we repeated the $\mathrm{I} S \mathrm{~A} \rightarrow \mathrm{A} \rightarrow \mathrm{s} \mathrm{A}$ protocol with a $2 \mathrm{~h}$ training period. A one-way ANOVA revealed a significant effect of condition $\left(F_{(3,20)}=69.005 ; p<\right.$ $0.001)$, but again a Bonferroni $t$ test found no significant effect of food on adaptation $(\mathrm{IsOA} \rightarrow \mathrm{IsOA}$ vs Sero $+\mathrm{IsOA} \rightarrow \mid \mathrm{soA})$.

associative) of learning based on their deficits in the different experimental protocols. The insulin pathway has a well known role in regulating C. elegans life span (Vanfleteren and Braeckman, 1999) and dauer formation (Fielenbach and Antebi, 2008). Insulin was first implicated in C. elegans associative learning with the isolation of the aho-2 mutant, a predicted null allele of the insulin homolog insulin-like 1 (ins-1), which displayed defective tracking to its cultivation temperature and food/starvation associative learning (Mohri et al., 2005; Kodama et al., 2006). Furthermore, ins-1 was also shown to play a role in salt starvation associative learning (Tomioka et al., 2006; Vellai et al., 2006), a $\mathrm{Cu}^{2+}$-diacetyl sensory integration paradigm (Jiu et al., 2010), and in forms of AWC olfactory associative learning (Chalasani et 

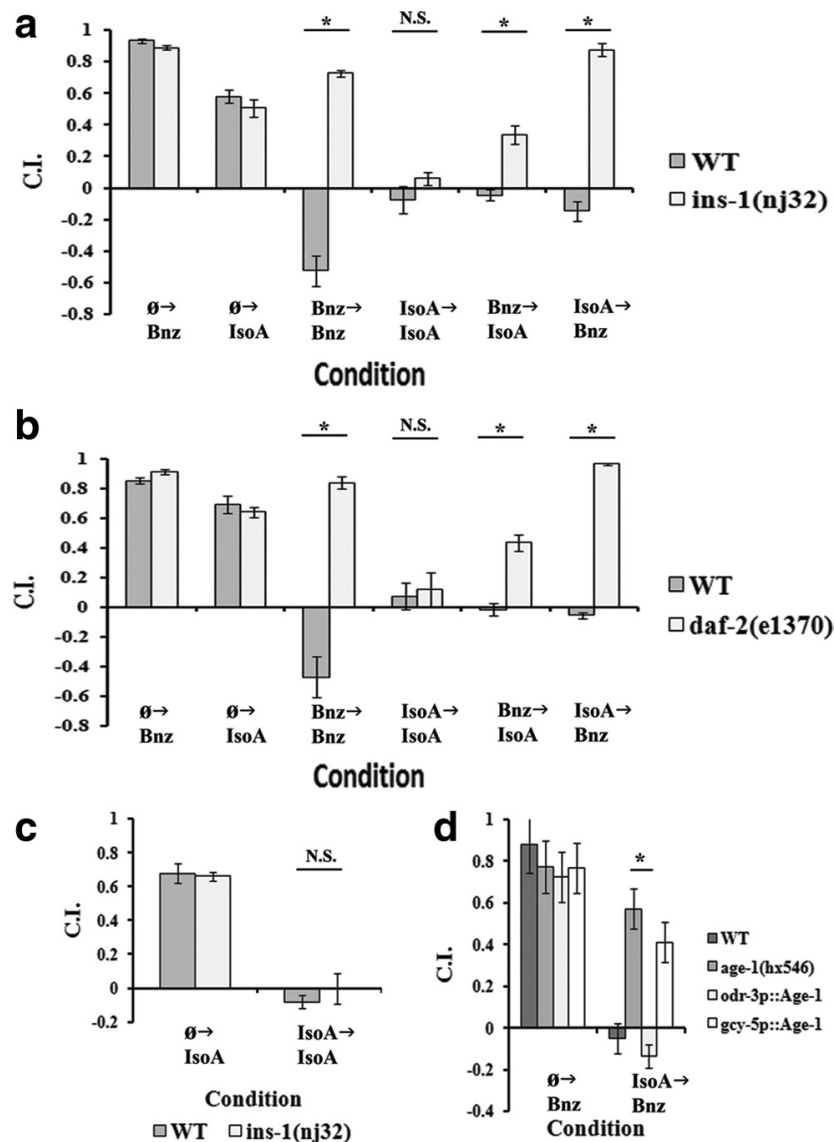

Figure 4. Insulin signaling is required for only the associative learning experimental protocols. For all panels, data shown are mean $\pm \mathrm{SEM}$, and an asterisk indicates significant difference between strains within condition ( ${ }^{*} p<0.05$ by Bonferroni's $t$ test), whereas N.S. indicates not significant. $n=6$ plates for all conditions. In $\boldsymbol{a}$ and $\boldsymbol{b}$, worms were tested in the following conditions from left to right: approach to Bnz after starvation ( $\emptyset \rightarrow B n z)$, approach to IsoA after starvation $(\emptyset \rightarrow \mid \mathrm{soA})$, approach to $\mathrm{Bnz}$ after Bnz training (Bnz $\rightarrow \mathrm{Bnz})$, approach to IsoA after IsoA training (IsoA $\rightarrow \mid s 0 A)$, approach to IsoA after Bnz training (Bnz $\rightarrow \mid s 0 A)$, and approach to $\mathrm{Bnz}$ after $2 \mathrm{~h}$ of $\mathrm{IsOA}$ training (IsoA $\rightarrow$ Bnz). For simplicity, the $2 \mathrm{~h}$ starvation only approach to $\mathrm{Bnz}$, which is not significantly different from that of the $1 \mathrm{~h}$ starvation approach, is omitted. $\boldsymbol{a}$, Comparison between WT and ins-1(nj32) mutant animals in starvation-only approach to Bnz and $\mathrm{IsO} \mathrm{A}$ and all four adaptation experimental protocols. A two-way ANOVA revealed a significant interaction between strain and condition $\left(F_{(5,60)}=56.02 ; p<0.001\right)$. A post hoc Bonferroni $t$ test revealed significant differences in $\mathrm{Bnz} \rightarrow \mathrm{Bnz}, \mathrm{Bnz} \rightarrow \mid \mathrm{so} \mathrm{A}$, and $\mathrm{Is} \mathrm{s} \rightarrow \mathrm{Bnz}$ adaptation, but not $\mathrm{Is} 0 \mathrm{~A} \rightarrow \mid \mathrm{s} 0 \mathrm{~A}$ adaptation, between wild-type and ins- 1 mutants. $\boldsymbol{b}$, Comparison between WT and daf-2(e1370) mutant animals in starvation-only approach to Bnz and IsoA and all four adaptation experimental protocols. A two-way ANOVA revealed a significant interaction between strain and condition $\left(F_{(5,60)}=53.12 ; p<0.001\right)$. Again, a post hoc Bonferroni $t$ test revealed significant differences in $\mathrm{Bnz} \rightarrow \mathrm{Bnz}, \mathrm{Bnz} \rightarrow \mathrm{IsOA}$, and $\mathrm{IsOA} \rightarrow \mathrm{Bnz}$ adaptation, but not IsoA $\rightarrow \mid$ soA adaptation, between wild-type and daf-2 mutants. $C$, Comparison between WT and ins-1(nj32) in the Iso $\mathrm{A} \rightarrow \mid$ IsoA permutation using a $2 \mathrm{~h}$ training period. A twoway ANOVA $\left(F_{(1,20)}=0.630 ; p=0.437\right)$ found no significant interaction between strain and

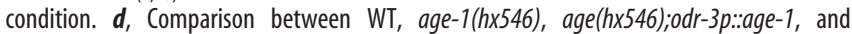
age-1(hx546); $g c y-5 p:: a g e-1$ in the Iso $A \rightarrow B n z$ protocol. The odr -3 and $g c y-5$ promoters drive expression primarily in the AWC and ASER neurons, respectively. A two-way ANOVA revealed a significant interaction between strain and condition $\left(F_{(3,40)}=22.888 ; p<0.001\right)$. A Bonferroni $t$ test found a significant difference after training between WT and age-1(hx546) and between age-1( $h \times 546$ ) and the odr-3p, but not $g c y-5 p$, rescue line. In addition, no significant difference could be found between $\mathrm{N} 2$ and the odr-3p rescue line, indicating that expression under the promoter is sufficient for a complete functional rescue of the phenotype.

al., 2010; Lin et al., 2010). By using a conditional allele of the $C$. elegans insulin receptor homolog abnormal dauer formation 2 (daf-2), our previous work (Lin et al., 2010) demonstrated that insulin signaling was only partially required during training, but
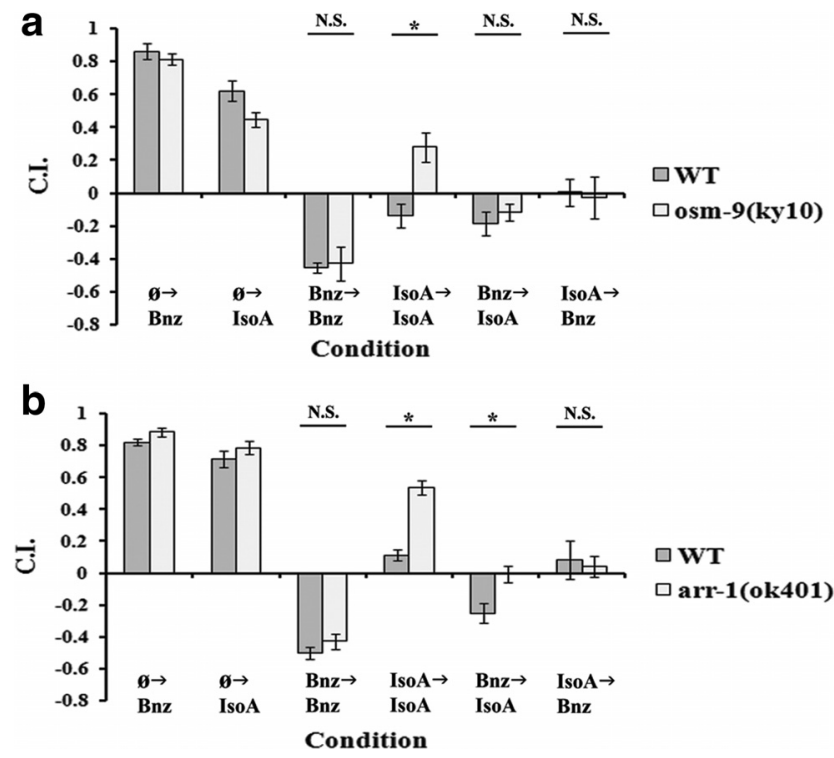

Figure 5. osm- 9 and arr- 1 are required for the nonassociative $\mathrm{I} S \mathrm{~A} \rightarrow \mathrm{I} 0 \mathrm{~A}$ learning, but only arr- 1 is also necessary in the associative $B n z \rightarrow \mid s 0 A$. For all panels, data shown are mean \pm $\mathrm{SEM}$, and an asterisk indicates significant difference between strains within condition $\left({ }^{*} p<\right.$ 0.05 by Bonferroni's $t$ test), whereas N.S. indicates not significant. $n=6$ plates for all conditions. In $\boldsymbol{a}$ and $\boldsymbol{b}$, conditions are laid out as described for Figure $4, a$ and $b$. $\boldsymbol{a}$, Comparison between WT and osm-9(ky10) mutants in starvation-only approach to Bnz and IsoA and all four adaptation experimental protocol. A two-way ANOVA revealed a significant interaction between strain and condition $\left(F_{(5,60)}=3.76 ; p<0.01\right)$. Bonferroni's corrections revealed a significant difference for only the IsoA $\rightarrow \mid$ soA protocol. $\boldsymbol{b}$, Comparison between WT and arr1(ok401) mutants in starvation-only approach to $\mathrm{Bnz}$ and Iso and all four adaptation experimental protocols. A two-way ANOVA revealed a significant interaction between strain and condition $\left(F_{(5,60)}=5.96 ; p<0.01\right)$. Bonferroni's corrections revealed a significant difference for the $\mathrm{Is} 0 \mathrm{~A} \rightarrow \mid \mathrm{s} 0 \mathrm{~A}$ and $\mathrm{Bnz} \rightarrow \mathrm{Is} 0 \mathrm{~A}$ experimental protocols.

absolutely essential during memory retrieval processes of $\mathrm{Bnz} \rightarrow \mathrm{Bnz}$ associative learning. We thus tested an ins-1 loss-offunction mutant in all four adaptation protocols (Fig. 4a). Consistent with our previous results, ins-1 (nj32) displayed wild-type chemotaxis to both odorants, but had a significant adaptation deficit in Bnz $\rightarrow$ Bnz learning. In addition, the results in Figure $4 a$ demonstrate that ins-1 function is necessary in both crossadaptations (Bnz $\rightarrow$ IsoA and IsoA $\rightarrow \mathrm{Bnz}$ ) but not in the Iso $A \rightarrow$ IsoA scenario. That is, ins- 1 dependence is entirely correlated with food and serotonin sensitivity. We have previously shown that a strain carrying a temperature-sensitive conditional allele of the insulin receptor, daf-2(e1370), demonstrates a complete adaptation deficit in Bnz $\rightarrow \mathrm{Bnz}$ adaptation when tested at the nonpermissive temperature (Lin et al., 2010). We therefore tested daf-2(e1370) in each of the adaptation protocols at the nonpermissive temperature (Fig. $4 b$ ). Once again, only the IsoA $\rightarrow$ IsoA protocol revealed adaptation indistinguishable from wild type, while defects in adaptation were apparent in all three other protocols. To again account for a possible effect of training period between IsoA $\rightarrow \operatorname{IsoA}(1 \mathrm{~h})$ and IsoA $\rightarrow \mathrm{Bnz}(2 \mathrm{~h})$, we tested ins-1 mutants in a $2 \mathrm{~h}$ training variant of the Iso $\mathrm{A} \rightarrow$ IsoA paradigm (Fig. $4 c$ ). The $2 \mathrm{~h}$ training version of the IsoA $\rightarrow$ IsoA protocol did not reveal an effect of ins-1 mutation, thus ruling out any effect of training period. These results support the notion that insulin signaling is essential in associative learning paradigms in which starvation is used as the unconditioned stimulus. In addition, the data argue that the IsoA $\rightarrow$ IsoA pathway is accessing a memory that is distinct from the other three experimental protocols and is not a form of associative learning. The work of 
Lin et al. (2010) suggested that function of the insulin pathway in the AWC sensory neuron itself was sufficient to rescue $\mathrm{Bnz} \rightarrow \mathrm{Bnz}$ associative learning in an insulin pathway mutant, age-1( $h \times 546)$, background. Similarly, we could completely rescue IsoA $\rightarrow \mathrm{Bnz}$ adaptation in an age-1 mutant with expression of age-1 in the AWC (Fig. 4d), despite the fact that worms were trained to a different odorant than in the Bnz $\rightarrow$ Bnz learning reported by Lin et al. (2010). These results strengthen the suggestion that Iso $\mathrm{A} \rightarrow \mathrm{Bnz}$ and $\mathrm{Bnz} \rightarrow \mathrm{Bnz}$ represent the same memory trace, one that is distinct from that sampled in the IsoA $\rightarrow$ IsoA protocol.

\section{Nonassociative IsoA learning requires osm-9 and arr-1}

In their initial description of the phenomenon of crossadaptation, Colbert and Bargmann (1995) tested mutations of osmotic avoidance defective-9 (osm-9) in the various experimental protocols. OSM-9 is a member of TRPV channels (Colbert et al., 1997) that functions by complexing with OCR-2 (Tobin et al., 2002) and is regulated by polyunsaturated fatty acids (KahnKirby et al., 2004). osm-9 has been shown to play a role in the function of ASH, the C. elegans polymodal nociception neuron, AWA chemosensation, the other olfactory sensory neuron pair mediating attractive olfaction and AWC adaptation (Colbert et al., 1997). The results of Colbert and Bargmann suggested osm9(ky10), a loss-of-function mutant, demonstrated deficits to both IsoA $\rightarrow$ IsoA and IsoA $\rightarrow$ Bnz adaptation (suggesting an IsoA acquisition defect) while providing contradictory assessments of the performance of $o s m-9(k y 10)$ in standard Bnz $\rightarrow$ Bnz adaptation [compare osm-9 Bnz $\rightarrow$ Bnz adaptation in the study by Colbert and Bargmann (1995), their Fig. 5A,C]. We thus revisited the role of osm-9 in olfactory adaptation by testing osm-9(ky10) in all scenarios (Fig. 5a). Consistent with the results of Colbert and Bargmann (1995), baseline AWC chemosensation was left unperturbed, and we found $o s m-9$ was required for IsoA $\rightarrow$ IsoA adaptation. Yet we were unable to find any significant perturbation of adaptation in the other three protocols in the osm-9 mutant. This result was the reciprocal of the insulin signaling mutants and thus provides a genetic double dissociation between the two forms of AWC olfactory plasticity. We confirmed, as shown previously, that osm-9 expression in the AWC was sufficient for rescue of the phenotype (data not shown). This implies osm-9 is specifically required in only nonassociative learning, while being dispensable for the insulin-dependent associative learning pathway that underlies adaptation in the other three experimental protocols.

The C. elegans arrestin homolog arrestin family-1 (arr-1) is also expressed in the AWC and has been reported to play a role in olfactory adaptation (Palmitessa et al., 2005). As before, we subjected the arr-1(ok401) deletion mutant to analysis in the four experimental protocols (Fig. $5 b$ ). arr-1 did not have a significant deficit in naive approach to either odorant, nor an adaptation deficit in the $\mathrm{Bnz} \rightarrow \mathrm{Bnz}$ and Iso $\mathrm{A} \rightarrow \mathrm{Bnz}$ experimental protocols. However, like osm-9, arr-1 mutation did reveal reduced adaptation in the IsoA $\rightarrow$ IsoA paradigm. In addition, unlike osm-9, arr- 1 mutation also significantly reduced $\mathrm{Bnz} \rightarrow$ IsoA adaptation relative to wild type, which suggests arr-1 is promiscuous between nonassociative and associative learning in mediating the decreased approach to IsoA.

\section{The cGMP-dependent kinase egl-4 mediates associative} memory formation

Work by the Bargmann and L'Etoile groups has highlighted a role for the cGMP-dependent kinase egg laying defective-4 (egl-4) in AWC Bnz olfactory adaptation (L'Etoile et al., 2002; O'Halloran
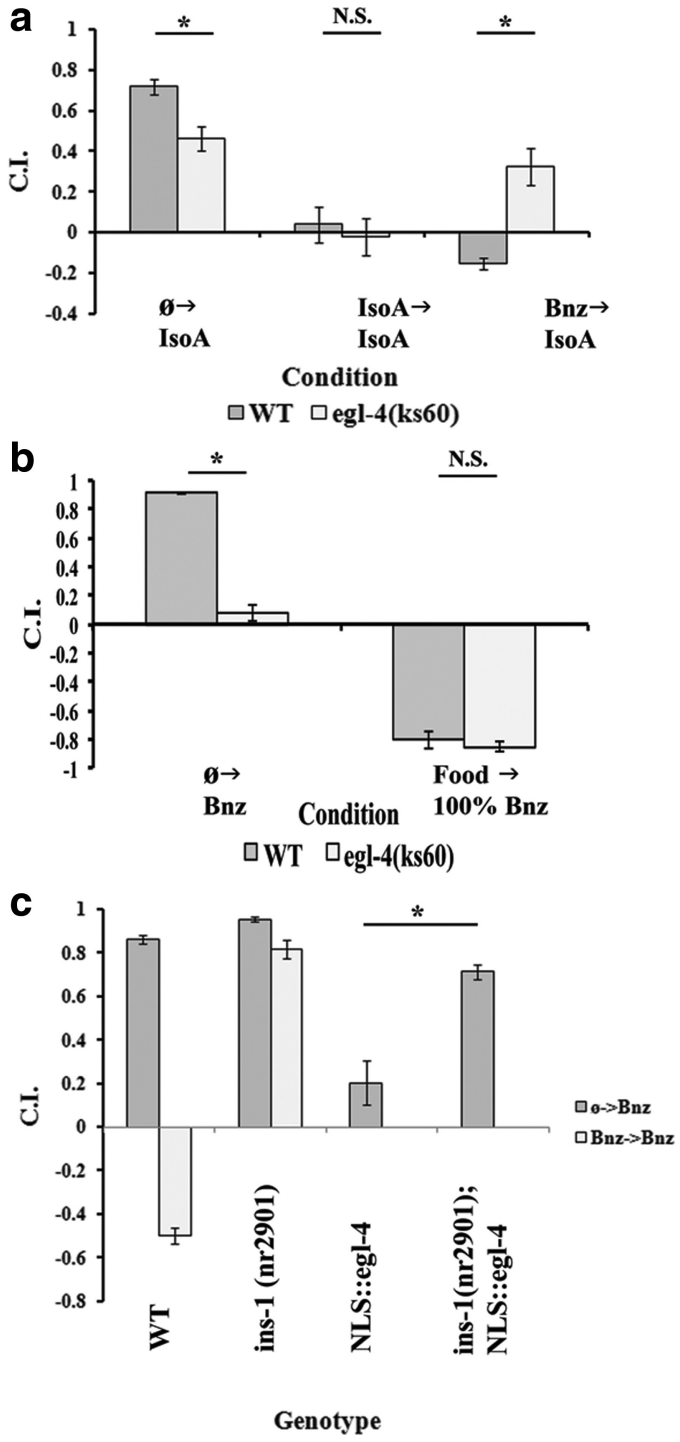

Figure 6. Insulin signaling is epistatic to egl-4, which is only required in associative learning For all panels, data shown are mean \pm SEM, and an asterisk indicates significant difference between strains within condition ( ${ }^{*} p<0.05$ by Bonferroni's $t$ test), whereas N.S. indicates not significant. $n=6$ plates for all conditions. $\boldsymbol{a}$, Comparison between wild type and egl-4(ks60) for approach to IsoA following either starvation only (left), training to IsoA (middle), or training to $\mathrm{Bnz}$ (right). A two-way ANOVA revealed a significant interaction between strain and condition $\left(F_{(2,30)}=14.221 ; p<0.001\right)$. A Bonferroni $t$ test indicated a significant difference between strains for both unconditioned approach and after training to Bnz, but not after training to IsoA. $\boldsymbol{b}$, Comparison of the responses of WT and egl-4(ks60) to 1\% Bnz after starvation (left), the standard Bnz retrieval stimulus used to test $\mathrm{Bnz} \rightarrow \mathrm{Bnz}$ and $\mathrm{lso} A \rightarrow \mathrm{Bnz}$, and $100 \%$ Bnz directly from maintenance conditions (right), the training stimulus used in $\mathrm{Bnz} \rightarrow \mathrm{Bnz}$ and $\mathrm{Bnz} \rightarrow \mathrm{Is} 0 \mathrm{~A}$. A two-way ANOVA revealed a significant interaction between strain and concentration $\left(F_{(1,20)}=81.73 ; p<0.001\right)$. A Bonferroni $t$ test revealed a significant difference between strains when tested at $1 \%$ Bnz but not $100 \%$ Bnz. c, Comparison of N2 and ins-1(nr2901) in their Bnz approach in naive Bnz US or Bnz $\rightarrow$ Bnz adapted states (2 left pairs) with the preadapted NLS::GFP.:EGL-4 in a N2 or ins-1(nr2901) background (2 rights columns). Groups involving training the strains with the constitutive allele are omitted, as they would have confounding sources of adaptation. A one-way ANOVA revealed a significant interaction between strain and condition $\left(F_{(5,30)}=122.790 ; p<0.001\right)$. Most important, a Bonferroni $t$ test indicated a significant difference between the N2 naive approach and that of the NLS::GFP.:EGL-4, but also between the NLS::GFP::EGL-4 and the ins-1(nr2901); NLS::GFP::EGL-4 double mutant.

et al., 2009; Lee et al., 2010). Intriguingly, O’Halloran et al. (2009) postulated that egl-4 functioned upstream of osm-9 in mediating long-term olfactory adaptation in the AWC sensory neuron because osm-9 mutation was unable to perturb nuclear localization 


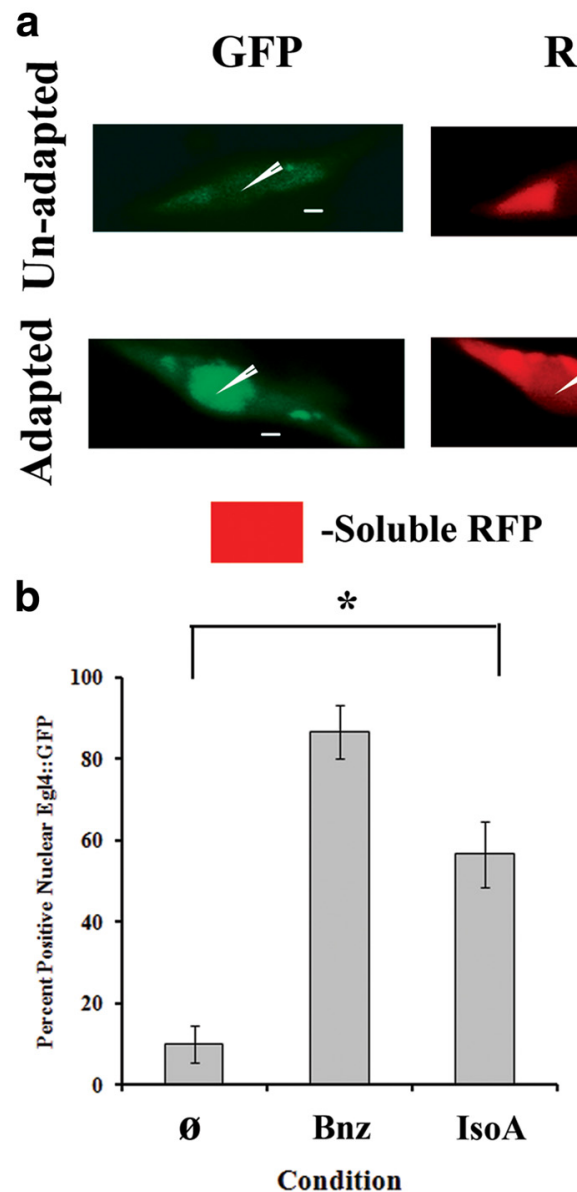

RFP

MERGE
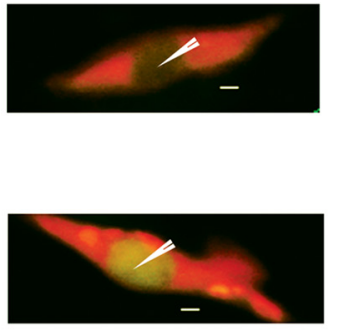

-GFP::EGL-4

C

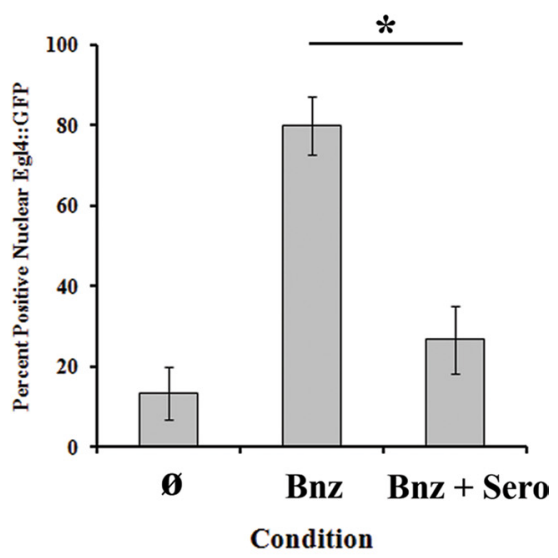

Figure 7. Nuclear accumulation of EGL-4 can be induced by IsoA and Bnz-induced nuclear localization can be blocked by serotonin. For $\boldsymbol{b}$ and $\boldsymbol{c}$, data shown are mean \pm SEM, and an asterisk indicates significant difference between strains within condition ( ${ }^{*} p<0.05$ by Bonferroni's $t$ test). Thirty worms were analyzed ( $n=6$ slides) for all conditions. $\boldsymbol{a}$, Representative images from confocal analysis of nuclear localization of EGL-4 (green) in the AWC neuron (red) after $2 \mathrm{~h}$ of starvation only (top panels) and training to Bnz (bottom panels) demonstrating the unadapted and adapted states, respectively. The arrowheads point into the AWC nucleus to highlight the difference in EGL-4 nuclear accumulation between the unadapted and adapted states. $\boldsymbol{b}$, Quantification of the IsoA induction of nuclear EGL-4. A one-way ANOVA revealed a significant effect of condition $\left(F_{(2,15)}=34.741 ; p<\right.$ 0.001 ), and a Bonferroni $t$ test revealed a significant difference between all conditions. $c$, Quantification of the effect of serotonin on obviating Bnz training. A one-way ANOVA revealed a significant effect of condition $\left(F_{(2,15)}=22.105 ; p<0.001\right)$. A Bonferroni $t$ test indicated a significant effect of serotonin on Bnz training (Bnz condition vs sero block), but not between US only and sero block conditions, highlighting that serotonin functions by preventing nuclear accumulation of EGL-4. strate the adaptation defect of the egl-4 mutation is specific to $\mathrm{Bnz} \rightarrow \mathrm{IsoA}$ and not IsoA $\rightarrow$ IsoA. However, such a result could also be due to an inability of the egl-4 mutants to sense the Bnz training stimulus used in the $\mathrm{Bnz} \rightarrow \mathrm{IsoA}$ protocol. To account for this possibility, we assayed for the approach of naive wild-type and naive egl-4 mutants to $1 \mu \mathrm{l}$ of $100 \% \mathrm{Bnz}$. We found no discernable difference between the behavior of either strain to the odorant (Fig. 6b). This suggests that the egl-4 mutant is able to sense the Bnz training stimulus and consequently that egl-4 plays a role in associative rather than nonassociative forms of AWC olfactory adaptation.

Experiments using a transgenic constitutively nuclear allele of egl-4 (NLS::GFP:: EGL-4) demonstrated that the nuclear accumulation of the protein was itself sufficient to phenocopy the conditioned response to Bnz (O'Halloran et al., 2009; Lee et al., 2010). The results thus far suggested that both the insulin signaling pathway and egl-4 function in the associative learning pathway. We therefore performed an epistasis test using the NLS::GFP::EGL-4 hypermorph, which places naive worms in a pseudoadapted state, and an ins-1 null allele, which eliminates adaptation (Fig. $6 c$ ). The data demonstrate that the mutant ins-1 allele is able to significantly rescue the naive $\mathrm{Bnz}$ approach of the NLS::GFP::EGL-4 transgenic to levels indistinguishable from the Bnz conditioned ins-1 mutant. Of note, we found that this was not a result of ins-1 mutation preventing nuclear localization of the EGL-4 transgene (data not shown). Thus, the ability of ins-1 mutation to obviate the pseudoadapted state implies that ins- 1 is epistatic to egl-4 in the associative learning pathway. of EGL-4, which is believed to mediate the latter's role in adaptation (O'Halloran et al., 2009; Lee et al., 2010). Yet it remains unclear from this work whether egl-4-mediated adaptation does indeed represent a form of habituation, upstream of osm-9, or is actually a form of associative learning.

We sought to clarify this issue by using our chemotactic assay to analyze the role of egl-4 in our two forms of learning. egl$4(k s 60)$ is a C-terminal deletion mutant just downstream of the kinase domain (Hirose et al., 2003), which is necessary in both chemotaxis and adaptation (L'Etoile et al., 2002). However, we found that mutation in egl-4 can also display significant deficits in naive chemotaxis to AWC-sensed odorants, which may be due to its regulation of the TAX-2/TAX-4 ion channels necessary for AWC chemotaxis (L'Etoile et al., 2002). While we found approach to Bnz was sufficiently compromised to prevent us from analysis of adaptation in the egl-4(ks60) mutant (Fig. 6b), its naive approach to IsoA was relatively unaffected. We thus analyzed egl-4(ks60) in the two experimental protocols that are dependent on IsoA-induced chemotaxis. The results in Figure $6 a$ demon-
EGL-4 nuclear localization can be induced by IsoA, and Bnz-induced nuclear localization is sensitive to serotonin during training

To further elucidate how egl-4 is involved in the three associative learning protocols, we used a transgenic line with a GFP-tagged EGL-4 and an RFP-labeled AWC (N2;odr-3p::gfp::egl-4;odr-1p::rfp), henceforth pyIS worms, to study its nuclear localization (O'Halloran et al., 2009). In line with our previous findings, IsoA training was also able to trigger the nuclear accumulation of EGL-4, albeit at a lower level than Bnz, although consistent with the weaker associative behavioral change seen with IsoA training (Fig. 7a,b). Most important, this validates a link between IsoA and the associative learning pathway predicted by our data. In addition, when training to Bnz was performed in the presence of exogenous serotonin, nuclear accumulation of EGL-4 was significantly impaired (Fig. 7c). This suggested that food/serotonin acts to block the formation of an associative memory trace by preventing the nuclear localization of EGL-4 through an unknown mechanism. 


\section{Discussion}

A number of unexpected, yet intriguing, implications can be elucidated from the results presented. Most surprising is the fact that only one (IsoA $\rightarrow$ IsoA) of the four adaptation protocols of IsoA and Bnz training and testing was insensitive to the presence of food or serotonin during the training period and hence a form of nonassociative learning that is not dependent of the pairing of odorant with a starvation unconditioned stimulus. This result was particularly unexpected because, while Iso $A \rightarrow$ Iso $A$ was nonassociative, the IsoA $\rightarrow$ Bnz protocol was associative, despite the fact that they use training to the same odorant and differ only in the retrieval stimulus used. This simple result has three profound inferences. First, training to IsoA must lead to the formation of at least two distinct memory traces, one associative and one nonassociative. Second, using Bnz or IsoA as a retrieval stimulus, following IsoA training, leads to a behavioral response that is preferentially dependent on either the associative or nonassociative memory trace, respectively. This represents, to our knowledge, the first demonstration in which the form of learning displayed after training to a single stimulus is a function of the retrieval stimulus used. Occam's razor led us to believe cross-adaptation in both directions (Iso $\rightarrow \mathrm{Bnz}$ and $\mathrm{Bnz} \rightarrow$ IsoA) occurred at the same step in adaptation and was due to a common mechanism (Atkinson-Leadbeater et al., 2004), the results indicate that the reciprocal crossadaptation is the result of two distinct unidirectional processes in the adaptation pathways (Fig. 8). That is, Iso $\mathrm{A} \rightarrow \mathrm{Bnz}$ cross-adaptation is a result of a mechanism distinct from $\mathrm{Bnz} \rightarrow$ IsoA crossadaptation. While the Iso $\mathrm{A} \rightarrow \mathrm{Bnz}$ cross occurs before the food/serotonin-sensitive step in adaptation, the reciprocal $\mathrm{Bnz} \rightarrow$ IsoA cross must come after it since both cross-adaptations are forms of associative learning and must pass through the food/serotonin-sensitive step. Furthermore, because $\mathrm{Bnz} \rightarrow$ IsoA adaptation is also dependent on insulin signaling, which we have shown has its most essential role in memory retrieval (Lin et al., 2010), the implication is that the $\mathrm{Bnz} \rightarrow$ IsoA cross-adaptation occurs downstream of insulin signaling during memory retrieval. In contrast, the Iso $\mathrm{A} \rightarrow \mathrm{Bnz}$ cross, which is also insulin dependent, is a result of processes during memory acquisition. Thus, this series of simple experiments has dramatically altered our conception of AWC olfactory learning.

It is significant that the majority of the processes involved in the integration of the CS and US in associative learning and in nonassociative memory formation ostensibly occurs within the AWC sensory neuron itself. Indeed, all the genes discussed in our

\section{Adaptation Model}

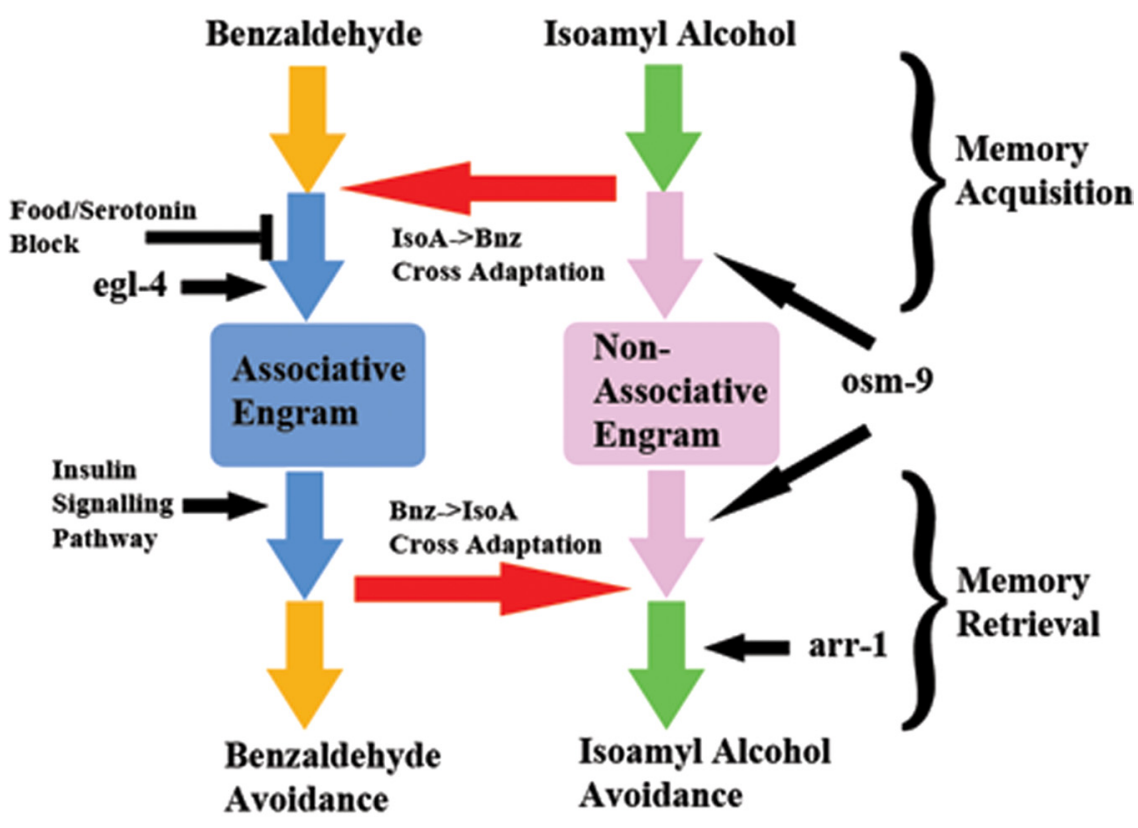

Associative learning core pathway

Non-associative learning specific pathway

-Cross Adaptation pathways

Figure 8. A model for two forms of learning in AWC olfactory plasticity. The model is laid out chronologically from top to bottom, with conditioned stimulus listed at the top and the behavioral responses (avoidance of either odor) listed at the bottom. The black arrows indicate location in the pathway where food/serotonin block or a given gene functions. Each odorant (Bnz or IsoA) begins by triggering a distinct set of processes based on each binding to its cognate chemoreceptor. For Bnz (start top left), this leads to the formation of the associative memory (left box) trace unless blocked by the presence of food/serotonin during food/serotoninsensitive step in the pathway. Furthermore, this process is dependent on EGL-4 function. However, IsoA (start top right) training results in the formation of two memory traces. The nonassociative memory (right box) through the adaptation process shown going down or the associative memory through the IsoA $\rightarrow$ Bnz unidirectional cross (top horizontal path) and then continuing through the associative learning-specific process that leads to the formation of the associative engram (left box). In this latter case the pathway from IsoA to the associative memory must similarly go through the food/serotonin-sensitive step and egl-4. osm- 9 is only required for the $\mathrm{IsO} \mathrm{A} \rightarrow \mathrm{Is} \mathrm{A}$ permutation, while arr- 1 has an additional role in $\mathrm{Bnz} \rightarrow \mathrm{IsOA}$ adaptation. This suggests osm- 9 determine whether osm- 9 functions before or after the nonassociative engram (right box). The ambiguity regarding whether osm- 9 functions in the acquisition or retrieval phase of the nonassociative memory is illustrated by the two arrows indicating its possible sites of action in the pathway. Intriguingly, the results for arr- 1 have the implication that associative and nonassociative memories may converge on similar molecules, and perhaps mechanism, to mediate the change in behavioral output (decreased attraction to IsoA) seen after learning. The nonassociative engram can result in only one possible behavioral output, the adapted response to IsoA when retrieved by that same stimulus (bottom right). In contrast, the associative trace leads to the conditioned response to both odorants by either going down the pathway with retrieval to Bnz (bottom left) or using the unidirectional $\rightarrow$ IsoA cross (bottom horizontal path) for retrieval to IsoA (bottom right). In either case, retrieval of the memory is a process that is insulin dependent. Insulin is depicted with its primary function in retrieval of the associative memory (black arrow), although Lin et al. (2010) suggest it also has a more minor role in associative acquisition. This function, also likely downstream of egl-4 from the results in Figure $6 c$ (placing it between egl-4 and the associative engram), is omitted for simplicity.

model are known to be expressed in and required within or act on, in the case of ins-1, the AWC to mediate olfactory adaptation (Colbert et al., 1997; L'Etoile et al., 2002; Palmitessa et al., 2005; Chalasani et al., 2010; Lin et al., 2010), lending support to the remarkable notion that the majority of processing occurs within the primary olfactory neuron. Indeed, this appears to be a common theme in a number of paradigms in C. elegans. For example, work by the Iino group has suggested that salt starvation associative learning requires insulin signaling within the salt-sensing ASE sensory neuron (Tomioka et al., 2006). Furthermore, food 
Table 1. Summary of the findings reported in this work

\begin{tabular}{|c|c|c|c|c|c|c|}
\hline & \multicolumn{4}{|c|}{ Testing odorant } & \multirow[b]{4}{*}{ Memory phase } & \multirow[b]{4}{*}{ Memory type } \\
\hline & $\mathrm{Bnz}$ & $\mathrm{Bnz}$ & IsoA & IsoA & & \\
\hline & \multicolumn{4}{|c|}{ Training odorant } & & \\
\hline & $\mathrm{Bnz}$ & IsoA & IsoA & $\mathrm{Bnz}$ & & \\
\hline \multicolumn{7}{|l|}{ Manipulation/strain } \\
\hline Food block & Yes & Yes & No & Yes & Acquisition & Associative \\
\hline Serotonin block & Yes & Yes & No & Yes & Acquisition & Associative \\
\hline ins-1 & Yes & Yes & No & Yes & Retrieval & Associative \\
\hline daf-2 & Yes & Yes & No & Yes & Retrieval & Associative \\
\hline osm-9 & No & No & Yes & No & Unknown & Nonassociative \\
\hline arr-1 & No & Yes & Yes & No & Retrieval & Both \\
\hline egl-4 & $\mathrm{N} / \mathrm{A}$ & Yes & No & N/A & Acquisition & Associative \\
\hline
\end{tabular}

Experimental protocol are listed on top horizontally, and strains and manipulations tested are listed in the left column. "Yes" indicates the manipulation tested (the presence of food or serotonin or a homozygous mutation) was able to significantly block adaptation relative to $\mathrm{N} 2$ adapted and tested to the same set of odorants in the standard condition (without the presence of food or serotonin), while " $\mathrm{No}$ " indicates the resulting adaptation were indistinguishable with or without manipulation. The right two columns indicate the assigned role for each manipulation in the phase of memory during which it acts (acquisition or retrieval) and the type of memory it is involved in (associative or nonassociative). Note that food, serotonin, and the insulin signaling mutants perturb exactly the opposite combination of the four adaptation protocols than the osm- 9 mutants, while the arr- 1 mutation in required for the $\mathrm{Bnz} \rightarrow \mathrm{IsOA}$ cross-adaptation in addition to the $\mathrm{IsO} \mathrm{A} \rightarrow \mathrm{IsOA}$ adaptation, which requires $05 \mathrm{sm}-9$. N/A indicates "not applicable" since these experimental protocols depend on chemotaxis to Bnz, which is compromised in the egl-4 mutant. Note that insulin is categorized by its primary role in memory retrieval, although in the study by Lin et al (2010) a more minor role in memory acquisition was also suggested.

starvation associative learning leads to a modulation of the temperature at which the temperature-sensing AFD primary sensory neurons (Kimura et al., 2004) and AWC neurons fire calcium transients (Biron et al., 2008; Kuhara et al., 2008). Similarly, Chalasani et al. (2010) have argued that in ins-1 mutants, AWC firing behavior after training is distinct from that seen in wild-type animals. Together, these findings suggests that information processing that is highly distributed over various substrates in more complex organisms is, in C. elegans, condensed to the level of the sensory neuron, which itself performs large amounts of processing, and a few interneurons (de Bono and Maricq, 2005).

Atkinson-Leadbeater et al. (2004) were able to use a strategy of using the four IsoA and Bnz adaptation experimental protocols to assign memory acquisition versus retrieval roles. Briefly, by demonstrating that the CB4856 line has a partial deficit in $\mathrm{Bnz} \rightarrow \mathrm{Bnz}$ but not $\mathrm{Bnz} \rightarrow \mathrm{IsoA}$ learning, their work suggested a Bnz-specific memory retrieval impairment due to an unmapped polymorphism. We believed a reverse genetic approach may be fruitful to dissociating memory acquisition from retrieval. Furthermore, the genetic analysis undertaken revealed a double dissociation between the nonassociative and associative forms of learning that suggest the presence of two distinct memory traces following training to IsoA (Table 1). Insulin signaling was required in mediating the $\mathrm{Bnz} \rightarrow \mathrm{Bnz}, \mathrm{Bnz} \rightarrow \mathrm{IsoA}$ and IsoA $\rightarrow \mathrm{Bnz}$ experimental protocols, thus exactly mirroring the pattern seen with the food and serotonin blocks. Similarly, egl-4 function was required in the $\mathrm{Bnz} \rightarrow \mathrm{IsoA}$ but not IsoA $\rightarrow$ IsoA permutation. These results suggest strongly that egl-4 and insulin signaling play a role in the formation and manifestation of associative learning while being dispensable for the nonassociative habituation. In contrast, the osm-9 mutants revealed the reciprocal pattern, with osm-9 being only required for the nonassociative IsoA $\rightarrow$ IsoA habituation while being dispensable for the associative experimental protocols. However, it remains unclear whether osm-9 functions in acquisition or retrieval of nonassociative memory. Most important, these mutations do not eliminate the sensation of the odorants, but rather the mutations block one of two different types of learning about these olfactory stimuli, all within the primary olfactory sensory neuron itself. Even more intriguing is the finding that arr-1 plays a role in both IsoA $\rightarrow$ IsoA and $\mathrm{Bnz} \rightarrow$ IsoA adaptations despite their reliance on distinct memory traces. This finding is particularly intriguing in light of our previous work (Morrison and van der Kooy, 2001) that demonstrated the AMPA-type glutamate receptor can play a role in both the nonassociative adaptation to the AWA-sensed odorant diacetyl and the associative pairing of diacetyl with the aversive unconditioned stimulus acetic acid. This suggests that distinct nonassociative and associative memories may nonetheless both use common downstream effectors (ARR-1) to generate a behavioral response (loss of IsoA attraction).

Our understanding of the AWC adaptation process using the four protocols was supplemented by additional experiments, most importantly, the demonstration that IsoA training can induce the nuclear localization of EGL-4. Given that we have shown that egl-4 is a component of the associative learning process, since it is required in $\mathrm{Bnz} \rightarrow$ IsoA but not IsoA $\rightarrow$ IsoA adaptation, this provides clear evidence that IsoA training can trigger the formation of the associative memory trace in addition to the nonassociative osm-9-dependent memory. Furthermore, serotonin can prevent the nuclear localization of EGL-4, which suggests that nuclear EGL-4 functions downstream of the food/serotoninsensitive step. The order of genes in the associative pathway was further deciphered by the experiment in Figure $6 c$. Our previous work (Lin et al., 2010) suggested the main function of insulin occurs during memory retrieval, and the work of Lee et al. (2010) suggested that egl-4 functions in memory acquisition. Briefly, Lee et al. (2010) demonstrated that the adaptation memory significantly outlasts the nuclear localization of EGL-4, and thus while its translocation may trigger formation of the engram, the physiological manifestation of memory, it is not the engram itself. Our result demonstrated that ins- 1 is epistatic to egl-4 and is therefore consistent with the role of egl-4 in memory acquisition and insulin in memory retrieval. All the data have been incorporated into a model of the adaptation pathways describing the formation of the memory traces and their activation to trigger a behavioral response (Fig. 8). While this model was initially purely conceptual, based on our dissociation of associative and nonassociative learning using the four adaptation protocols, our mutational analysis has enabled us to translate it into a partial genetic description of the adaptation processes.

In the study by O'Halloran et al. (2009), the authors assayed nuclear localization of EGL-4 in various mutant backgrounds to ask whether specific mutations acted upstream or downstream. When the authors discovered that osm-9 mutation had no discernable effect on EGL-4 nuclear localization, this was taken to imply that OSM-9 functioned downstream of EGL-4. However, our data suggest that this result is explained by OSM-9 and EGL-4 functioning in two different forms of learning, nonassociative and associative, respectively. This reinterpretation is also consistent with the fact that other groups have reported no benzaldehyde adaptation deficit in osm-9 [Colbert and Bargmann (1995), their Fig. 5A] and that loss of osm-9 was able to suppress a chemotactic deficit to IsoA, but not to Bnz, in a tax-6 loss of function mutant (Kuhara et al., 2002). In contrast, this latter result can be easily incorporated into our model by postulating a role for tax-6 between IsoA sensation and the IsoA $\rightarrow$ Bnz crossover (top right of model). Loss of tax-6 places worms in a pseudoadapted state, as suggested by Kuhara et al. (2002), which activates both pathways. However, while osm-9 mutation can rescue approach to IsoA by blocking the nonassociative pathway, it cannot block the Iso $\mathrm{A} \rightarrow \mathrm{Bnz}$ associative learning pathway and hence is unable to rescue the approach to Bnz of the tax-6 mutant. Our work there- 
fore demonstrates that, although $\mathrm{Bnz}$ and IsoA act upon the AWC and can cross-adapt, they nonetheless retain the ability to activate distinct molecular pathways, which results in a unique interplay of associative and nonassociative learning processes.

\section{References}

Atkinson-Leadbeater K, Nuttley WM, van der Kooy D (2004) A genetic dissociation of learning and recall in Caenorhabditis elegans. Behav Neurosci 118:1206-1213.

Bargmann CI, Horvitz HR (1991) Chemosensory neurons with overlapping functions direct chemotaxis to multiple chemicals in C. elegans. Neuron 7:729-742.

Bargmann CI, Hartwieg E, Horvitz HR (1993) Odorant-selective genes and neurons mediate olfaction in C. elegans. Cell 74:515-527.

Biron D, Wasserman S, Thomas JH, Samuel AD, Sengupta P (2008) An olfactory neuron responds stochastically to temperature and modulates Caenorhabditis elegans thermotactic behavior. Proc Natl Acad Sci U S A 105:11002-11007.

Chalasani SH, Kato S, Albrecht DR, Nakagawa T, Abbott LF, Bargmann CI (2010) Neuropeptide feedback modifies odor-evoked dynamics in Caenorhabditis elegans olfactory neurons. Nat Neurosci 13:615-621.

Colbert HA, Bargmann CI (1995) Odorant-specific adaptation pathways generate olfactory plasticity in C. elegans. Neuron 14:803-812.

Colbert HA, Smith TL, Bargmann CI (1997) OSM-9, a novel protein with structural similarity to channels, is required for olfaction, mechanosensation and olfactory adaptation in Caenorhabditis elegans. J Neurosci 17:8259-8269.

de Bono M, Maricq AV (2005) Neuronal substrates of complex behaviors in C. elegans. Annu Rev Neurosci 28:451-501.

Fielenbach N, Antebi A (2008) C. elegans dauer formation and the molecular basis of plasticity. Genes Dev 22:2149-2165.

Giles AC, Rankin CH (2009) Behavioral and genetic characterization of habituation using Caenorhabditis elegans. Neurobiol Learn Mem 92:139-146.

Groves PM, Thompson RF (1970) Habituation: a dual process theory. Psychol Rev 77:419-450.

Ha HI, Hendricks M, Shen Y, Gabel CV, Fang-Yen C, Qin Y, Colón-Ramos D, Shen K, Samuel AD, Zhang Y (2010) Functional organization of a neural network for aversive olfactory learning in Caenorhabditis elegans. Neuron 68:1173-1186.

Hedgecock EM, Russell RL (1975) Normal and mutant thermotaxis in the nematode Caenorhabditis elegans. Proc Natl Acad Sci USA 72: 4061-4065.

Hirose T, Nakano Y, Nagamatsu Y, Misumi T, Ohta H, Ohshima Y (2003) Cyclic GMP-dependent protein kinase EGL-4 controls body size and lifespan in C. elegans. Development 130:1089-1099.

Jiu YM, Yue Y, Yang S, Liu L, Yu JW, Wu ZX, Xu T (2010) Insulin-like signaling pathway functions in integrative response to an olfactory and gustatory stimuli in Caenorhabditis elegans. Protein Cell 1:75-81.

Kahn-Kirby AH, Dantzker JL, Apicella AJ, Schafer WR, Browse J, Bargmann CI, Watts JL (2004) Specific polyunsaturated fatty acids drive TRPVdependent sensory signaling in vivo. Cell 119:889-900.

Kimura KD, Miyawaki A, Matsumoto K, Mori I (2004) The C. elegans thermosensory neuron AFD responds to warming. Curr Biol 14:1291-1295.

Kodama E, Kuhara A, Mohri-Shiomi A, Kimura KD, Okumura M, Tomioka M, Iino Y, Mori I (2006) Insulin-like signaling and the neural circuit for integrative behavior in C. elegans. Genes Dev 20:2955-2960.

Kuhara A, Inada H, Katsura I, Mori I (2002) Negative regulation and gain control of sensory neurons by the C. elegans calcineurin TAX-6. Neuron 33:751-763.

Kuhara A, Okumura M, Kimata T, Tanizawa Y, Takano R, Kimura KD, Inada $\mathrm{H}$, Matsumoto K, Mori I (2008) Temperature sensing by an olfactory neuron in a circuit controlling behavior of C. elegans. Science 320:803-807.

Lee JI, O'Halloran DM, Eastham-Anderson J, Juang BT, Kaye JA, Scott Hamilton O, Lesch B, Goga A, L'Etoile ND (2010) Nuclear entry of a cGMP- dependent protein kinase converts transient into long lasting olfactory adaptation. Proc Natl Acad Sci U S A 107:6016-6021.

L'Etoile ND, Coburn CM, Eastham J, Kistler A, Gallegos G, Bargmann CI (2002) The cyclic GMP-dependent protein kinase EGL-4 regulates olfactory adaptation in C. elegans. Neuron 36:1079-1089.

Levitan IB, Kaczmarek LK (1991) Learning and memory. In: The neuron: cell and molecular biology, pp 395-423. New York: Oxford UP.

Lin CH, Tomioka M, Pereira S, Sellings L, Iino Y, van der Kooy D (2010) Insulin signaling plays a dual role in Caenorhabditis elegans memory acquisition and memory retrieval. J Neurosci 30:8001-8011.

Luedtke S, O'Connor V, Holden-Dye L, Walker RJ (2010) The regulation of feeding and metabolism in response to food deprivation in Caenorhabditis elegans. Invert Neurosci 10:63-76.

Mohri A, Kodama E, Kimura KD, Koike M, Mizuno T, Mori I (2005) Genetic control of temperature preference in the nematode Caenorhabditis elegans. Genetics 169:1437-1450.

Mori I, Ohshima Y (1995) Neural regulation of thermotaxis in Caenorhabditis elegans. Nature 376:344-348.

Morrison GE, van der Kooy D (2001) A mutation in the AMPA-type glutamate receptor, glr-1, blocks olfactory associative and non-associative learning in Caenorhabditis elegans. Behav Neurosci 115:640-649.

Morrison GE, Wen JY, Runciman S, van der Kooy D (1999) Olfactory associative learning in Caenorhabditis elegans is impaired in $l r n-1$ and $l r n-2$ mutants. Behav Neurosci 113:358-367.

Nuttley WM, Atkinson-Leadbeater KP, van der Kooy D (2002) Serotonin mediates food-odor associative learning in the nematode Caenorhabditis elegans. Proc Natl Acad Sci U S A 99:12449-12454.

O'Halloran DM, Altshuler-Keylin S, Lee JI, L'Etoile ND (2009) Regulators of AWC-mediated olfactory plasticity in Caenorhabditis elegans. PLoS Genet 5:e1000761.

Palmitessa A, Hess HA, Bany IA, Kim YM, Koelle MR, Benovic JL (2005) Caenorhabditis elegans arrestin regulates neural $\mathrm{G}$ protein signaling and olfactory adaptation and recovery. J Biol Chem 280:24649-24662.

Ryder EF, Robakiewicz P (2001) Statistics for the molecular biologist: group comparisons. Curr Protoc Mol Biol Appendix 3:Appendix 3I.

Saeki S, Yamamoto M, Iino Y (2001) Plasticity of chemotaxis revealed by paired presentation of a chemoattractant and starvation in the nematode Caenorhabditis elegans. J Exp Biol 204:1757-1764.

Tobin D, Madsen D, Kahn-Kirby A, Peckol E, Moulder G, Barstead R, Maricq A, Bargmann C (2002) Combinatorial expression of TRPV channel proteins defines their sensory functions and subcellular localizations in $C$. elegans neurons. Neuron 35:307-318.

Tomioka M, Adachi T, Suzuki H, Kunitomo H, Schafer WR, Iino Y (2006) The insulin/PI 3-kinase pathway regulates salt chemotaxis learning in Caenorhabditis elegans. Neuron 51:613-625.

Torayama I, Ishihara T, Katsura I (2007) Caenorhabditis elegans integrates the signals of butanone and food to enhance chemotaxis to butanone. J Neurosci 27:741-750.

Troemel ER, Kimmel BE, Bargmann CI (1997) Reprogramming chemotaxis responses: sensory neurons define olfactory preferences in C. elegans. Cell 91:161-169.

Tsunozaki M, Chalasani SH, Bargmann CI (2008) A behavioral switch: cGMP and PKC signaling in olfactory neurons reverses odor preference in C. elegans. Neuron 59:959-971.

Vanfleteren JR, Braeckman BP (1999) Mechanisms of life span determination in C. elegans. Neurobiol Aging 20:487-502.

Vellai T, McCulloch D, Gems D, Kovács AL (2006) Effects of sex and insulin/insulin-like growth factor-1 signaling on performance in an associative learning paradigm in Caenorhabditis elegans. Genetics 174:309-316

Wen JY, Kumar N, Morrison G, Rambaldini G, Runciman S, Rousseau J, van der Kooy D (1997) Mutations that prevent associative learning in $C$. elegans. Behav Neurosci 111:354-368.

Wes PD, Bargmann CI (2001) C. elegans odor discrimination requires asymmetric diversity in olfactory neurons. Nature 410:698-701.

Zhang Y, Lu H, Bargmann CI (2005) Pathogenic bacteria induce aversive olfactory learning in Caenorhabditis elegans. Nature 438:179-184. 